Funding and Support: This research was supported by an unrestricted grant to the Casey Eye Institute from Research to Prevent Blindness, New York, NY. Dr Yeh has received support from the Heed Ophthalmic Foundation and the Ronald G Michels foundation.

Eye (2010) 24, 1625-1627; doi:10.1038/eye.2010.111; published online 13 August 2010

\section{Sir, \\ Patient, surgical, and lens-related factors, and their association with Hydroview intraocular lens opacification}

We read with interest Rimmer et al's letter ${ }^{1}$ published in January suggesting a higher incidence of Hydroview lens (Bausch and Lomb) calcification in those implanted between late 2000 and mid-2001. The main cause of opacification was thought to be silicone in the lens packaging. ${ }^{2,3}$ As the packaging did not change between 1997 and 2001, ${ }^{2}$ Rimmer et al's observations suggest that other aetiological factors might be involved.

A study set up at Bristol assessed the impact of Hydroview opacification on vision (Central and South Bristol Research Ethics Committee Ref 05/Q2006/163). ${ }^{4}$ We re-evaluated the data from this study to investigate the associations of opacification with patient, surgical, and lens-related factors. Data were gathered through a review of medical records, patient interview, and examination.

Data from 215 patients who had Hydroview lenses implanted in Bristol were analysed: 125 had clear

Table 1 Association between lens opacification and ocular pathology

\begin{tabular}{|c|c|c|c|c|}
\hline & \multicolumn{4}{|c|}{ IOL opacified } \\
\hline & $\begin{array}{c}\text { No } \\
(\mathrm{n}=125)\end{array}$ & $\begin{array}{c}\text { Yes } \\
(\mathrm{n}=89)\end{array}$ & $\begin{array}{c}\text { Total } \\
(\mathrm{n}=214)\end{array}$ & P-value \\
\hline \multicolumn{5}{|c|}{ Diabetic retinopathy in the study eye } \\
\hline No & $117(58.8 \%)$ & $82(41.2 \%)$ & $199(100 \%)$ & \multirow{2}{*}{0.89} \\
\hline Yes & $8(53.3 \%)$ & $7(46.7 \%)$ & $15(100 \%)$ & \\
\hline \multicolumn{5}{|c|}{ Glaucoma in the study eye } \\
\hline No & $109(57.4 \%)$ & $81(42.6 \%)$ & $190(100 \%)$ & \multirow{2}{*}{0.52} \\
\hline Yes & $16(66.7 \%)$ & $8(33.3 \%)$ & $24(100 \%)$ & \\
\hline \multicolumn{5}{|c|}{ Uveitis in the study eye } \\
\hline No & $123(58.3 \%)$ & $88(41.7 \%)$ & $211(100 \%)$ & \multirow{2}{*}{$>0.99$} \\
\hline Yes & 2 & 1 & $3^{\mathrm{b}}$ & \\
\hline \multicolumn{5}{|c|}{ Vitrectomy in the study eye } \\
\hline No & $119(59.5 \%)$ & $81(40.5 \%)$ & $200(100 \%)$ & \multirow{2}{*}{0.35} \\
\hline Yes & $6(42.9 \%)$ & $8(57.1 \%)$ & $14(100 \%)$ & \\
\hline
\end{tabular}

lenses, 89 had evidence of opacification, and 1 lens could not be assessed. No statistically significant association was demonstrated between opacification and any of the patient- (Tables 1 and 2) or surgery-related factors (Table 3 ) tested. However, this is likely to be a result of the small number of patients at subgroup analysis level.

There was a marked association of opacification with certain runs of consecutive lens serial numbers (Table 4). Serial numbers relate only to the order in which the lenses are manufactured and are sequential. Our study will have selection bias, as all patients had surgery over a similar time period and those symptomatic would be more likely to enrol. There will be a high number of certain runs of serial numbers and a high number of opacified lenses. However, variation in the proportion of lenses opacified (3-79\%) across the serial numbers tested strongly suggests an association between opacity and certain runs of serial numbers.

Although these data must be viewed with respect to the context, ie, as a retrospective observation rather than as prospective evidence, they do question the supposition that the sole cause for opacification was the

Table 2 Association between lens opacification and systemic pathology

\begin{tabular}{|c|c|c|c|c|}
\hline & IOL of & cified & & \\
\hline & No & Yes & Total & P-value \\
\hline Hypertensi & & & & \\
\hline No & $51(60.7 \%)$ & $33(39.3 \%)$ & $84(100 \%)$ & \\
\hline Yes & $74(56.9 \%)$ & $56(43.1 \%)$ & $130(100 \%)$ & 0.684 \\
\hline Total & $125(58.4 \%)$ & $89(41.6 \%)$ & $214(100 \%)$ & \\
\hline Ischaemic 1 & art disease & & & \\
\hline No & $100(61.0 \%)$ & $64(39.0 \%)$ & $164(100 \%)$ & \\
\hline Yes & $25(50.0 \%)$ & $25(50.0 \%)$ & $50(100 \%)$ & 0.225 \\
\hline Total & $125(58.4 \%)$ & $89(41.6 \%)$ & $214(100 \%)$ & \\
\hline Cerebrovas & lar event & & & \\
\hline No & $120(58.3 \%)$ & $86(41.7 \%)$ & $206(100 \%)$ & \\
\hline Yes & $5(62.5 \%)$ & $3(37.5 \%)$ & $8(100 \%)$ & 1.000 \\
\hline Total & $125(58.4 \%)$ & $89(41.6 \%)$ & $214(100 \%)$ & \\
\hline Inflammato & condition & & & \\
\hline No & $122(58.1 \%)$ & $88(41.9 \%)$ & $210(100 \%)$ & \\
\hline Yes & $3(75.0 \%)$ & $1(25.0 \%)$ & $4(100 \%)$ & 0.867 \\
\hline Total & $125(58.4 \%)$ & $89(41.6 \%)$ & $214(100 \%)$ & \\
\hline Cancer & & & & \\
\hline No & $121(58.5 \%)$ & $86(41.5 \%)$ & $207(100 \%)$ & \\
\hline Yes & $4(57.1 \%)$ & $3(42.9 \%)$ & $7(100 \%)$ & 1.000 \\
\hline Total & $125(58.4 \%)$ & $89(41.6 \%)$ & $214(100 \%)$ & \\
\hline Gender & & & & \\
\hline Male & $48(55.8 \%)$ & $38(44.2 \%)$ & $86(100 \%)$ & \\
\hline Female & $77(60.2 \%)$ & $51(39.8 \%)$ & $128(100 \%)$ & 0.624 \\
\hline Total & $125(58.4 \%)$ & $89(41.6 \%)$ & $214(100 \%)$ & \\
\hline
\end{tabular}

${ }^{a} \chi^{2}$-test was performed with continuity correction asymptotic significance two-sided. 
Table 3 Association between lens opacification and surgical factors

\begin{tabular}{|c|c|c|c|c|}
\hline & \multicolumn{2}{|c|}{ IOL opacified } & \multirow[b]{2}{*}{ Total } & \multirow[b]{2}{*}{ P-value ${ }^{\mathrm{a}}$} \\
\hline & No & Yes & & \\
\hline \multicolumn{5}{|l|}{ Surgery type } \\
\hline Routine & $121(58.2 \%)$ & $87(41.8 \%)$ & $208(100 \%)$ & \multirow{3}{*}{$>0.99$} \\
\hline Combined & 3 & 2 & $5^{b}$ & \\
\hline & $(n=124)$ & $(n=89)$ & $(n=213)$ & \\
\hline \multicolumn{5}{|c|}{ Intra-operational complications } \\
\hline No & $121(59.0 \%)$ & $84(41.0 \%)$ & $205(100 \%)$ & \multirow[b]{2}{*}{$>0.99$} \\
\hline Yes & $\begin{array}{c}4 \\
(n=125)\end{array}$ & $\begin{array}{c}3 \\
(n=87)\end{array}$ & $\begin{array}{c}7^{\mathrm{b}} \\
(n=212)\end{array}$ & \\
\hline \multicolumn{5}{|c|}{ Complications post-operation } \\
\hline No & $114(59.1 \%)$ & $79(40.9 \%)$ & $193(100 \%)$ & \multirow[b]{2}{*}{$>0.99$} \\
\hline Yes & $\begin{array}{l}11(57.9 \%) \\
(n=125)\end{array}$ & $\begin{array}{c}8(42.1 \%) \\
(n=87)\end{array}$ & $\begin{array}{l}19(100 \%) \\
(n=212)\end{array}$ & \\
\hline \multicolumn{5}{|l|}{ Viscoelastic } \\
\hline Provisc & $54(60.7 \%)$ & $35(39.3 \%)$ & $89(100 \%)$ & \multirow[b]{2}{*}{0.88} \\
\hline Other & $\begin{array}{c}10(66.7 \%) \\
(n=64)\end{array}$ & $\begin{array}{l}5(33.3 \%) \\
(n=40)\end{array}$ & $\begin{array}{l}15(100 \%) \\
(n=104)\end{array}$ & \\
\hline \multicolumn{5}{|l|}{ Anaesthesia } \\
\hline General & $11(40.7 \%)$ & $16(59.3 \%)$ & $27(100 \%)$ & \\
\hline Local & $\begin{array}{c}113(61.1 \%) \\
(n=124)\end{array}$ & $\begin{array}{c}72(38.9 \%) \\
(n=88)\end{array}$ & $\begin{array}{c}185(100 \%) \\
(n=212)\end{array}$ & 0.07 \\
\hline
\end{tabular}

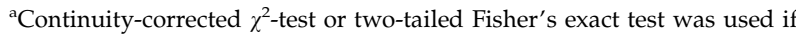
frequencies were small.

${ }^{\mathrm{b}}$ Too few for percentage calculation.

Table 4 Association between lens opacification and serial number

\begin{tabular}{|c|c|c|c|}
\hline $\begin{array}{l}\text { Serial } \\
\text { number }\end{array}$ & $\begin{array}{l}\text { Number of } \\
\text { eyes } \\
\text { examined }\end{array}$ & $\begin{array}{l}\text { Number of } \\
\text { eyes } \\
\text { opacified }\end{array}$ & $\begin{array}{c}\text { Opacification } \\
\text { rate } \\
(95 \% \mathrm{CI})\end{array}$ \\
\hline 4JAA00-4N9999 & 2 & 0 & \multirow{7}{*}{$3 \%(0-17 \%)$} \\
\hline 4PAA00-4T9999 & 4 & 0 & \\
\hline 4UAA00-4Y9999 & 11 & 1 & \\
\hline 4ZAA00-439999 & 0 & 0 & \\
\hline 44AA00-489999 & $1^{\mathrm{a}}$ & 0 & \\
\hline 49AA00-5D9999 & 2 & 0 & \\
\hline 5EAA00-5I9999 & 10 & 0 & \\
\hline 5JAA00-5N9999 & 25 & 1 & $4 \%(0-20 \%)$ \\
\hline 5PAA00-5T9999 & 24 & 2 & $8 \%(1-27 \%)$ \\
\hline 5UAA00-5Y9999 & 70 & 55 & $79 \%(67-88 \%)$ \\
\hline 5ZAA00-539999 & 35 & 19 & $54 \%(37-71 \%)$ \\
\hline 54AA00-589999 & 26 & 8 & \multirow{2}{*}{$33 \%(17-53 \%)$} \\
\hline 59AA00-6D9999 & 4 & 2 & \\
\hline Total & $214^{\mathrm{a}}$ & 88 & $41 \%(35-48 \%)$ \\
\hline
\end{tabular}

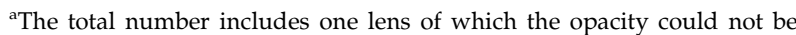
assessed and also another lens of which serial number was unknown.

silicon gasket. The findings support a manufacturerelated cause over a patient- or surgical-related cause for opacification. Although no conclusions can be drawn about the nature of this unknown aetiological factor, our data pinpoint it within a narrow time frame of manufacture.

\section{Conflict of interest}

The authors declare no conflict of interest.

\section{Acknowledgements}

Bausch and Lomb kindly gave financial support to this study to cover patient care.

\section{References}

1 Rimmer T, Hawkesworth N, Kirkpatrick N, Price N, Manners R, Ursell P. Calcification of Hydroview lenses implanted in the United Kingdom during 2000 and 2001. Eye (Lond) 2010; 24(1): 199-200.

2 Medicines and Healthcare Products Regulatory Agency. $H R A$ Report MDA/2004/015-Bausch \& Lomb HYDROVIEW H60M Intraocular Lens 2004.

3 Dorey MW, Brownstein S, Hill VE, Mathew B, Botton G, Kertes PJ et al. Proposed pathogenesis for the delayed postoperative opacification of the hydroview hydrogel intraocular lens. Am J Ophthalmol 2003; 135(5): 591-598.

4 Blundell MS, Mayer EJ, Knox Cartwright NE, Hunt LP, Tole DM, Dick AD. The effect on visual function of Hydroview intraocular lens opacification: A cross-sectional study. Eye (Lond) 2010. E-pub ahead of print 11 June 2010.

MSJ Blundell ${ }^{1}$, EJ Mayer ${ }^{2}$, NK Cartwright ${ }^{2}$, LP Hunt ${ }^{3}$, DM Tole ${ }^{2}$ and AD Dick ${ }^{1}$

${ }^{1}$ Academic Unit of Ophthalmology, Department of Clinical Sciences at South Bristol, University of Bristol, Bristol, UK

${ }^{2}$ Department of Ophthalmology, University Hospitals of Bristol NHS Foundation Trust, Bristol, UK ${ }^{3}$ Institute of Child life and Health, Department of Clinical Sciences at South Bristol, University of Bristol, Bristol, UK

E-mail: msjb1@doctors.org.uk

Eye (2010) 24, 1627-1628; doi:10.1038/eye.2010.97; published online 9 July 2010

\section{Sir, Effect of lyophilization on the in vitro biological activity of bevacizumab}

Intravitreal bevacizumab has been effective for vascular endothelial growth factor (VEGF)-mediated diseases of the retina and choroids. ${ }^{1,2,3}$ However, repeated injections may be required. An alternative mode of administration would be a biodegradable intravitreal implant ${ }^{4}$ of lyophilized bevacizumab, which has not been previously reported.

In an effort to assess the viability of a biodegradable intravitreal implant of lyophilized bevacizumab, we 